\title{
On matrix geometry
}

\author{
Harold Steinacker* \\ Fakultät für Physik, Universität Wien \\ Boltzmanngasse 5, A-1090 Wien, Austria \\ E-mail: harold.steinackereunivie.ac.at
}

The foundations of matrix geometry are discussed, which provides the basis for recent progress on the effective geometry and gravity in Yang-Mills matrix models. Basic examples lead to a notion of embedded noncommutative spaces (branes) with emergent Riemannian geometry. This class of configurations turns out to be preserved under small deformations, and is therefore appropriate for matrix models. The relation with spectral geometry is discussed. A possible realization of sufficiently generic 4-dimensional geometries as noncommutative branes in $D=10$ matrix models is sketched.

Corfu Summer Institute on Elementary Particles and Physics - Workshop on Non Commutative Field Theory and Gravity,

September 8-12, 2010

Corfu Greece

\footnotetext{
${ }^{*}$ Speaker.
} 


\section{Introduction}

One of the most fascinating ideas in recent years is the proposal that matrix models of YangMills type, in particular certain models which have been put forward in string theory $[1,2]$, may provide a description for the quantum structure of space-time and geometry. The beauty of the proposal lies in the simplicity of these models, whose structure is essentially

$$
S=\operatorname{Tr}\left[X^{a}, X^{b}\right]\left[X^{a^{\prime}}, X^{b^{\prime}}\right] g_{a a^{\prime}} g_{b b^{\prime}}+\text { fermions. }
$$

Here $X^{a}, a=1, \ldots, D$ are a set of hermitian matrices, and we restrict ourselves to the case of Euclidean signature with $g_{a b}=\delta_{a b}$ in this article. No notion of differential geometry and classical space-time whatsoever is used in this action. The geometrical structures arise in a certain "semiclassical limit", in terms of solutions of these models. The aim of this article is to clarify the scope and the mathematical description of this "emergent" geometry.

Simple examples of such matrix geometries, notably the fuzzy sphere $S_{N}^{2}$ or more general quantized homogeneous spaces including the Moyal-Weyl quantum plane $\mathbb{R}_{\theta}^{2 n}$, have been studied in great detail. However in order to describe the general geometries required for gravity, one cannot rely on the special group-theoretical structure of these simple examples. This obstacle was removed recently by realizing $[3,4]$ that there is a sufficiently large class of matrix geometries with generic geometry, which can play the role of space-time (possibly with extra dimensions) in YangMills matrix models. The key to understand their geometry is to realize that these generic matrix geometries should be considered as embedded noncommutative $(N C)$ spaces resp. NC branes $\mathscr{M} \subset$ $\mathbb{R}^{D}$. The effective geometry is very clear in the "semi-classical limit" of the matrix geometry, where commutators are replaced by Poisson brackets. As we will recall below, $\mathscr{M}$ then inherits the pullback metric $g_{\mu \nu}$ of $\mathbb{R}^{D}$, which combines with the Poisson (or symplectic) structure $\theta^{\mu v}(x)$ to form an effective metric $G_{\mu v}(x)$, much like the open string metric in string theory.

However, the description of matrix geometries in terms of a semi-classical limit is based on certain assumptions, and is may seem desirable to have a more precise characterization of "matrix geometries". This is possible in the simple examples mentioned above, introducing e.g. a differential calculus and using ideas of noncommutative differential geometry. The standard realization of these structures for the homogeneous spaces $[5,6,7]$ however relies on the special group-theoretical structure. Moreover, it turns out that this differential calculus is essentially that of the ambient space $\mathbb{R}^{D}$, and does not know about the intrinsic geometry of the fuzzy space. One might consider to use Connes NC differential calculus, which however is not naturally adapted to fuzzy geometries (e.g. there is an issue with chirality). Instead, we will follow an important lesson from Yang-Mills matrix models: there is no need for any additional structure, the models contain all the ingredients required for physics. Our task is merely to extract this information without mathematical prejudice. This is the strategy adopted here.

In this article, various aspects of matrix geometry arising in Yang-Mills matrix models and their mathematical description will be discussed. We first recall some examples of finite matrix geometries, described by finite-dimensional matrix algebras $\mathscr{A}=\operatorname{Mat}(\mathrm{N}, \mathbb{C})$. This includes a series of very clear and well-known examples such as the fuzzy sphere $S_{N}^{2}$, which can be considered for any $N \in \mathbb{N}$. However matrix geometries are much more general, and also cover singular geometries 
such as intersecting branes. Transitions between different topologies are conceivable, and physically very interesting. Therefore the meaning of matrix geometry and even topology can in general only be approximate, since for small $N$ there can be no way to exactly separate and characterize them. The appropriate concept is that of an effective or "emergent" geometry, which is valid within a certain range of energies. This is entirely sufficient for any physical application and in fact to be expected, since the Planck scale provides a natural limitation for geometry. Our task is therefore to find an appropriate and useful description of generic matrix geometries, where mathematical axioms on the geometry are replaced by estimates on the validity of certain effective descriptions. We will discuss the appropriate tools for this description here, delegating the estimates for future work.

\section{Examples of matrix geometries}

\subsection{Prototype: the fuzzy sphere}

The fuzzy sphere $S_{N}^{2}[5,8]$ is a quantization resp. matrix approximation of the usual sphere $S^{2}$, with a cutoff in the angular momentum. We first note that the algebra of functions on the ordinary sphere can be generated by the coordinate functions $x^{a}$ of $\mathbb{R}^{3}$ modulo the relation $\sum_{a=1}^{3} x^{a} x^{a}=1$. The fuzzy sphere $S_{N}^{2}$ is a non-commutative space defined in terms of three $N \times N$ hermitian matrices $X^{a}, a=1,2,3$ subject to the relations

$$
\left[X^{a}, X^{b}\right]=\frac{i}{\sqrt{C_{N}}} \varepsilon^{a b c} X^{c}, \quad \sum_{a=1}^{3} X^{a} X^{a}=1
$$

where $C_{N}=\frac{1}{4}\left(N^{2}-1\right)$ is the value of the quadratic Casimir of $\mathfrak{s u}(2)$ on $\mathbb{C}^{N}$. They are realized by the generators of the $N$-dimensional representation $(N)$ of $\mathfrak{s u}(2)$. The matrices $X^{a}$ should be interpreted as quantized embedding functions in the Euclidean space $\mathbb{R}^{3}$,

$$
X^{a} \sim x^{a}: \quad S^{2} \hookrightarrow \mathbb{R}^{3}
$$

They generate an algebra $\mathscr{A} \cong \operatorname{Mat}(\mathrm{N}, \mathbb{C})$, which should be viewed as quantized algebra of functions on the symplectic space $\left(S^{2}, \omega_{N}\right)$ where $\omega_{N}$ is the canonical $S U(2)$-invariant symplectic form on $S^{2}$ with $\int \omega_{N}=2 \pi N$. The best way to see this is to decompose $\mathscr{A}$ into irreps under the adjoint action of $S U(2)$, which is obtained from

$$
\begin{aligned}
S_{N}^{2} \cong(N) \otimes(\bar{N}) & =(1) \oplus(3) \oplus \ldots \oplus(2 N-1) \\
& =\left\{\hat{Y}_{0}^{0}\right\} \oplus \ldots \oplus\left\{\hat{Y}_{m}^{N-1}\right\} .
\end{aligned}
$$

This provides the definition of the fuzzy spherical harmonics $\hat{Y}_{m}^{l}$, and defines the quantization map

$$
\begin{aligned}
\mathscr{I}: \quad \mathscr{C}\left(S^{2}\right) & \rightarrow \mathscr{A}=\operatorname{Mat}(\mathrm{N}, \mathbb{C}) \\
Y_{m}^{l} & \mapsto\left\{\begin{array}{cc}
\hat{Y}_{m}^{l}, & l<N \\
0, & l \geq N
\end{array}\right.
\end{aligned}
$$

It follows easily that $\mathscr{I}\left(i\left\{x^{a}, x^{b}\right\}\right)=\left[X^{a}, X^{b}\right]$ where $\{$,$\} denotes the Poisson brackets correspond-$ ing to the symplectic form $\omega_{N}=\frac{N}{2} \varepsilon_{a b c} x^{a} d x^{b} d x^{c}$ on $S^{2}$. Together with the fact that $\mathscr{I}(f g) \rightarrow$ 
$\mathscr{I}(f) \mathscr{I}(g)$ for $N \rightarrow \infty$ (which is not hard to prove), $\mathscr{I}(i\{f, g\}) \approx[\mathscr{I}(f), \mathscr{I}(g)]$ follows. This means that $S_{N}^{2}$ is the quantization of $\left(S^{2}, \omega_{N}\right)$.

Moreover, there is a natural Laplace operator on $S_{N}^{2}$ defined as

$$
\square=\left[X^{a},\left[X^{b}, .\right]\right] \delta_{a b}
$$

which is invariant under $S O(3)$. Its spectrum coincides with the spectrum of the classical Laplace operator on $S^{2}$ up to the cutoff, and the eigenvectors are given by the fuzzy spherical harmonics $\hat{Y}_{m}^{l}$.

In this special example, (2.3) allows to construct a a series of embeddings

$$
\mathscr{A}_{N} \subset \mathscr{A}_{N+1} \subset \ldots
$$

with norm-preserving embedding maps. This allows to recover the classical sphere by taking the inductive limit. While this is a very nice structure, we do not want to rely on the existence of such series of embeddings, for reasons explained below.

\subsection{Other examples}

A straightforward generalization of the fuzzy sphere leads to the fuzzy complex projective space $\mathbb{C} P_{N}^{n-1}$, which is defined in terms of hermitian matrices $X^{a}, a=1,2, \ldots, n^{2}-1$ subject to the relations

$$
\left[X^{a}, X^{b}\right]=\frac{i}{\sqrt{C_{N}^{\prime}}} f_{c}^{a b} X^{c}, \quad d_{a b}^{c} X^{a} X^{b}=D_{N} X^{c}, \quad X_{a} X^{a}=1
$$

(adopting a sum convention). Here $f_{c}^{a b}$ are the structure constants of $\mathfrak{s u}(n), d^{a b c}$ is the totally symmetric invariant tensor, and $C_{N}^{\prime}, D_{N}$ are group-theoretical constants which are not needed here. These relations are realized by the generators of $\mathfrak{s u}(n)$ acting on irreducible representations $\mathbb{C}^{d_{N}}$ with highest weight $(N, 0, \ldots, 0)$ or $(0,0, \ldots, N)$. Again, the matrices $X^{a}$ should be interpreted as quantized embedding functions in the Euclidean space $\mathfrak{s u}(n) \cong \mathbb{R}^{n^{2}-1}$,

$$
X^{a} \sim x^{a}: \quad \mathbb{C} P^{n-1} \hookrightarrow \mathbb{R}^{n^{2}-1} .
$$

They generate an algebra $\mathscr{A} \cong \operatorname{Mat}\left(\mathrm{d}_{N}, \mathbb{C}\right)$, which should be viewed as quantized algebra of functions on the symplectic space $\left(\mathbb{C} P^{n-1}, N \omega\right)$ where $\omega$ is the canonical $S U(n)$-invariant symplectic form on $\mathbb{C} P^{n-1}$. It is easy to write down a quantization map analogous to (2.4),

$$
\mathscr{I}: \quad \mathscr{C}\left(\mathbb{C} P^{n-1}\right) \rightarrow \mathscr{A}
$$

using the decomposition of $\mathscr{A}$ into irreps of $\mathfrak{s u}(n)$. Again, there is a natural Laplace operator on $\mathbb{C} P_{N}^{n-1}$ defined as in (2.5) whose spectrum coincides with the classical one up to the cutoff. A similar construction can be given for any coadjoint orbit of a compact Lie group.

The fuzzy torus $T_{\theta}^{2}$ can be defined in terms of clock- and shift operators $U, V$ acting on $\mathbb{C}^{N}$ with relations $U V=q V U$ for $q^{N}=1$, with $U^{N}=V^{N}=1$. However one can also view it as embedded noncommutative space in $\mathbb{R}^{4}$, by defining 4 hermitian matrices $U=X^{1}+i X^{2}, V=X^{3}+i X^{4}$ which satisfy the relations

$$
\begin{aligned}
\left(X^{1}\right)^{2}+\left(X^{2}\right)^{2} & =1=\left(X^{3}\right)^{2}+\left(X^{4}\right)^{2} \\
\left(X^{1}+i X^{2}\right)\left(X^{3}+i X^{4}\right) & =q\left(X^{3}+i X^{4}\right)\left(X^{1}+i X^{2}\right) .
\end{aligned}
$$


They can again be viewed as embedding maps

$$
X^{a} \sim x^{a}: \quad T^{2} \hookrightarrow \mathbb{R}^{4} .
$$

These matrices generate the algebra $\mathscr{A} \cong \operatorname{Mat}(\mathrm{N}, \mathbb{C})$, which using the decomposition into irreps under $U(1) \times U(1)$ can be viewed as quantization of the function algebra $\mathscr{C}\left(T^{2}\right)$ on the symplectic space $\left(T^{2}, \omega_{N}\right)$. The spectrum of the matrix Laplacian (2.5) approximately coincides with the classical case below the cutoff.

Finally, the Moyal-Weyl quantum plane $\mathbb{R}_{\theta}^{2 n}$ is defined in terms of $2 n$ (infinite-dimensional) hermitian matrices $X^{a} \in \mathscr{L}(\mathscr{H})$ subject to the relations

$$
\left[X^{\mu}, X^{v}\right]=i \theta^{\mu v} 1
$$

where $\theta^{\mu \nu}=-\theta^{v \mu} \in \mathbb{R}$. Here $\mathscr{H}$ is a separable Hilbert space. This generates the (n-dimensional) Heisenberg algebra $\mathscr{A}$ (or some suitable refinement of it, ignoring operator-technical subtleties here), which can be viewed as quantization of the algebra of functions on $\mathbb{R}^{2 n}$ using e.g. the Weyl quantization map ${ }^{1}$. Of course, the matrices $X^{\mu}$ should be viewed as quantizations of the classical coordinate functions $X^{\mu} \sim x^{\mu}: \mathbb{R}^{2 n} \rightarrow \mathbb{R}^{2 n}$. Again the Laplacian (2.5) coincides with the classical one, for the effective metric specified in (4.6).

This concludes our brief exhibition of matrix geometries, whose geometry is obvious because of their symmetry. We will learn below how to generalize them for generic geometries, and how to systematically extract their geometry without using this symmetry. Besides these and other nice examples, there are also more exotic and singular spaces that can be modeled by matrices, such as intersecting spaces, stacks of spaces, etc.

\subsection{Lessons and cautions}

We draw the following general lessons from the above examples:

- The algebra $\mathscr{A}=\mathscr{L}(\mathscr{H})$ of linear operators on $\mathscr{H}$ should be viewed as quantization of the algebra of functions on some symplectic space $(\mathscr{M}, \omega)$. However as abstract algebra, $\mathscr{A}$ carries no geometrical information, not even the dimension or the topology of the corresponding space. $\operatorname{dim}(\mathscr{H})$ merely counts the number of "quantum cells", more precisely it measures the volume via the semi-classical relation (cf. (4.1))

$$
\int \frac{\omega^{n}}{n !} f \sim(2 \pi)^{n} \operatorname{Tr} \mathscr{I}(f)
$$

- The geometrical information is encoded in the specific matrices $X^{a}$, which should be interpreted as embedding functions

$$
X^{a} \sim x^{a}: \mathscr{M} \hookrightarrow \mathbb{R}^{D}
$$

They encode the embedding geometry, which is contained e.g. in the matrix Laplacian (2.5). We will learn below how to extract this more directly. The Poisson resp. symplectic structure is encoded in their commutation relations. In this way, even finite-dimensional matrices can describe various geometries to a high precision.

\footnotetext{
${ }^{1}$ which in turn is defined in terms of plane waves i.e. irreducible representations of the translation group.
} 
- In some sense, every non-degenerate and "regular" fuzzy space locally looks like the quantization of some Poisson manifold, in particular like some Moyal-Weyl quantum plane $\mathbb{R}_{\theta}^{2 n}$. The algebra of functions on $\mathbb{R}_{\theta}^{2 n}$ is infinite-dimensional only because its volume is infinite. For example, $\mathbb{C} P_{N}^{n}$ can be viewed as particular compactification of $\mathbb{R}_{\theta}^{2 n}$.

This leads to the idea that generic geometries can be described similarly as embedded noncommutative spaces in matrix models, interpreting the matrices $X^{a}$ as quantized embedding maps $X^{a} \sim x^{a}: \mathscr{M} \hookrightarrow \mathbb{R}^{D}$. However, some cautionary remarks on matrix geometries are in order.

The problem of identifying the geometry corresponding to some given configuration $\left\{X^{a}\right\}$ in the matrix model is clearly hard ${ }^{2}$, since general matrices do not necessarily admit a geometrical interpretation. There is not even a notion of dimension at this level of generality. In fact matrix models can describe much more general situations, such as multiple submanifolds ("branes"), intersecting branes, manifolds suspended between branes, etc., essentially the whole zoo of string theory. Each of these are very interesting and should be treated separately. Therefore we have to make some simplifying assumptions, and focus on the simplest case of classical submanifolds (and possibly stacks of coinciding branes.). Indeed, there is a large class of configurations which clearly have such a geometrical interpretation. For example, we will show in section 5 how to realize a large class of generic $4 \mathrm{~d}$ geometries through such matrix geometries.

A sharp separation between admissible and non-admissible matrix geometries would in fact be inappropriate in the context of matrix model, whose main merit is the definition of quantization in terms of an integral over the space of all matrices,

$$
Z=\int d X^{a} e^{-S[X]}
$$

and similarly for correlation functions. The ultimate aim is to show that the dominant contributions to this integral correspond to matrix configurations which have a geometrical meaning and are relevant to physics. However, the integral is over all possible matrices, including geometries with different dimensions and topologies. It is therefore clear that such a geometric notion can only be approximate or "emergent".

Finally, we want to address the issue of finite-dimensional versus infinite-dimensional matrix algebras. Imagine that our space-time was fuzzy, with an area quantization characterized by the scale $\Lambda_{\mathrm{NC}}$ (one may expect $\Lambda_{\mathrm{NC}} \approx \Lambda_{\text {Planck }}$ ), and perhaps even compact of size $R$ (e.g. with the topology of $T^{4}$ ). Then there would be only finitely many "quantum cells", and the geometry should be modeled by some finite $N$-dimensional (matrix) algebra. No experiment on earth, not even at CERN, can directly access the Planck scale, and all measurements about the geometry could be in perfect agreement with such a model in terms of a finite matrix geometry. Therefore the limit $N \rightarrow \infty$ is not essential for local physics, however there must be a large "separation of scales". Let $\Lambda_{\text {cosm }} \sim 1 / R$ some cosmological scale. Then as long as $\Lambda_{\text {cosm }} \ll \Lambda_{\mathrm{NC}}$ and $\Lambda_{\text {phys }} \ll \Lambda_{\mathrm{NC}}$ where $\Lambda_{\text {phys }}$ is the maximal available energy for experiments, then a description in terms of finite matrix geometries should be perfectly adequate. The effective geometry would be arguably the same if the spectrum of the corresponding fuzzy Dirac or Laplace operator approximately coincides with the continuum case up to energies of order $\Lambda_{\mathrm{NC}}$. There is no obvious requirement above that scale.

\footnotetext{
${ }^{2} \mathrm{~A}$ priori one does not have a sequence of matrices as in (2.6).
} 


\section{Spectral matrix geometry}

We want to understand more generally such "matrix geometries", described by a number of hermitian matrices $X^{a} \in \mathscr{A}=\mathscr{L}(\mathscr{H})$. Here $\mathscr{H}$ is a finite-dimensional or infinite-dimensional (separable) Hilbert space.

From an algebraic point of view, such matrix algebras are quite boring and in a sense trivial. In fact, Wedderburns theorem implies that the algebra generated by finite-dimensional hermitian matrices is always the product of simple matrix algebras. However, the point is that even simple matrix algebras can describe non-trivial geometries, as demonstrated by the above examples. It is the additional structure provided by the specific embedding matrices $X^{a}$ which makes such matrix geometries interesting and non-trivial.

One way to extract geometrical information from a space $\mathscr{M}$ which naturally generalizes to the noncommutative setting is via spectral geometry. In the classical case, one can consider the heat kernel expansion of the Laplacian $\Delta_{g}$ of a compact Riemannian manifolds $(\mathscr{M}, g)$ [9],

$$
\operatorname{Tr} e^{-\alpha \Delta_{g}}=\sum_{n \geq 0} \alpha^{(n-d) / 2} \int_{\mathscr{M}} d^{d} x \sqrt{|g|} a_{n}(x) .
$$

The Seeley-de Witt coefficients $a_{n}(x)$ of this asymptotic expansion are determined by the intrinsic geometry of $\mathscr{M}$, e.g. $a_{2} \sim-\frac{R[g]}{6}$ where $R[g]$ is the curvature scalar. This provides physically valuable information on $\mathscr{M}$, and describes the one-loop effective action. In particular, the leading term allows to compute the number of eigenvalues below some cutoff,

$$
\mathscr{N}_{\Delta}(\Lambda):=\#\left\{\mu^{2} \in \operatorname{spec} \Delta ; \mu^{2} \leq \Lambda^{2}\right\} .
$$

dropping the subscript $g$ of the Laplacian. One obtains Weyls famous asymptotic formula

$$
\mathscr{N}_{\Delta}(\Lambda) \sim c_{d} \operatorname{vol} \mathscr{M} \Lambda^{d}, \quad c_{d}=\frac{\operatorname{vol} S^{d-1}}{d(2 \pi)^{d}} .
$$

In particular, the (spectral) dimension $d$ of $\mathscr{M}$ can be extracted the from the asymptotic density of the eigenvalues of $\Delta_{g}$. However, although the spectrum of $\Delta_{g}$ contains a lot of information on the geometry, it does not quite determine it uniquely, and there are inequivalent but isospectral manifolds ${ }^{3}$.

Now consider the spectral geometry of fuzzy spaces in more detail. In the finite-dimensional case, the asymptotic density of eigenvalues strictly speaking vanishes, which would give the naive conclusion that fuzzy spaces (and all finite matrix geometries) have spectral dimension zero. Of course this completely misses the point. The proper definition of a spectral dimension in the fuzzy case with Laplacian $\square$ should be something like

$$
\mathscr{N}_{\square}(\Lambda) \sim c_{d} \operatorname{vol} \mathscr{M} \Lambda^{d} \quad \text { for } \Lambda \leq \Lambda_{\max }
$$

where $\Lambda_{\max }$ is the cutoff of the spectrum. This is of course a bit hard to make precise, but the idea is clear. Similarly, the information about the geometry of $\mathscr{M}$ is encoded in the spectrum of its

\footnotetext{
${ }^{3}$ One way to close this gap is to consider spectral triples associated to Dirac operators [10]. In the matrix model, the geometrical information will be extracted more directly using the symplectic structure and the embedding defined by the matrices $X^{a}$.
} 
Laplacian or Dirac operator below its cutoff. Such a cutoff is in fact essential to obtain meaningful Seeley-de Witt coefficients in the noncommutative case, see [11]. Thus if spec $\square$ has a clear enough asymptotics for $\Lambda \leq \Lambda_{\max }$ and approximately coincides with $\operatorname{spec} \Delta_{g}$ for some classical manifold $(\mathscr{M}, g)$ for $\Lambda \leq \Lambda_{\max }$, then its spectral geometry is that of $\mathscr{M}$.

To proceed, we need to specify a Laplacian for matrix geometries. Here the (Yang-Mills) matrix model provides a natural choice: For any given background configuration in the matrix model defined by $D$ hermitian matrices $X^{a}$, there is a natural matrix Laplace operator ${ }^{4}$

$$
\square=\left[X^{a},\left[X^{b}, .\right]\right] \delta_{a b}
$$

which is a (formally) hermitian operator on $\mathscr{A}$. We can study its spectrum and the distribution of eigenvalues. As we will explain below, this Laplacian governs the fluctuations in the matrix model, and therefore encodes its effective geometry. Hence if there is a classical geometry which approximates the matrix background $X^{a}$ up to some scale $\Lambda_{\mathrm{NC}}$, the spectrum of its canonical (LeviCivita) Laplacian $\Delta_{g}$ must approximately coincide with the spectrum of $\square$, up to some possible cutoff $\Lambda$. In particular, there should be a map between classical functions and NC functions

$$
\begin{aligned}
\mathscr{I}: \quad \mathscr{C}_{\Lambda}(\mathscr{M}) & \rightarrow \mathscr{A} \subset \operatorname{Mat}(\infty, \mathbb{C}) \\
f(x) & \mapsto F
\end{aligned}
$$

which approximately intertwines the Laplacians $\mathscr{I}\left(\Delta_{g} f\right) \approx \square(\mathscr{I}(f))$. Here $\mathscr{C}_{\Lambda}(\mathscr{M})$ denotes the space of functions on $\mathscr{M}$ whose eigenvalues are bounded by $\Lambda$, and $\mathscr{I}$ should be injective. The fuzzy sphere is an example where the matrix Laplacian precisely matches the classical Laplacian up to the cutoff. Its special symmetry is not essential here.

\section{Embedded noncommutative spaces and semi-classical limit.}

Although the idea of spectral geometry is clear and appropriate, it is very hard in practice to extract information on the metric from the spectrum. It would be much nicer to have a more direct handle on the geometry. This can indeed be achieved, assuming that the matrix configuration can be understood as quantization of an approximate classical symplectic manifold $\left(\mathscr{M}, \theta^{\mu v}\right)$. We can then take advantage of the noncommutative structure of the algebra encoded in the commutators, and interpret commutators as quantization of the Poisson structure on $\mathscr{M}$. In particular, the matrices $X^{a}$ will be interpreted as quantized embedding functions. This makes the framework of matrix model much more accessible than the geometry of abstract NC spaces.

Quantization of Poisson manifolds. The quantization of a Poisson (or symplectic) structure on $\mathscr{M}$ is given by a quantization map (generalizing (3.6)) such that

$$
\begin{aligned}
\mathscr{I}: \quad \mathscr{C}_{\Lambda}(\mathscr{M}) & \rightarrow \mathscr{A} \subset \operatorname{Mat}(\infty, \mathbb{C}) \\
f(x) & \mapsto F \\
f g & \mapsto F G+O(\theta), \quad\{f, g\} \mapsto-i[F, G]+O\left(\theta^{2}\right) .
\end{aligned}
$$

\footnotetext{
${ }^{4}$ This operator arises e.g. as equation of motion for the Yang-Mills matrix model. There is also a natural matrix Dirac operator $\not D \Psi=\Gamma_{a}\left[X^{a}, \Psi\right]$ where $\Gamma_{a}$ generates the Clifford algebra of $S O(D)$. However we will not discuss it here.
} 
Here $\theta$ encodes the scale of the Poisson tensor $\theta^{\mu v}=\left\{x^{\mu}, x^{v}\right\}$ in some local coordinates, and $O\left(\theta^{2}\right)$ stands for higher-order correction terms (which are unavoidable). This will allow to explicitly understand the geometry encoded in the matrix model background $X^{a}$, as explained below. In any case, it is clear that $\theta^{\mu v}$ - if it exists in nature - must play some dynamical physical role, which remains to be clarified.

The bottom line will be that any configurations in the matrix model which correspond to "almost-commutative" geometries can be related to this underlying classical space using (4.1). We can then talk about the semi-classical limit of the matrix model background. This means that every matrix $F$ will be replaced by its classical pre-image $\mathscr{I}^{-1}(F)=: f$, and commutators will be replaced by Poisson brackets. This allows to use the tools of classical differential geometry, and provides the leading approximation of the geometry. However one can go beyond this semiclassical limit, by defining an associative product on $\mathscr{C}(\mathscr{M})$ via

$$
f \star g:=\mathscr{I}^{-1}(\mathscr{I}(f) \mathscr{I}(g))
$$

This allows to systematically compute higher-order corrections of the NC case in the language of classical functions and geometry. The matrix model action (and any action in NC field theory) can then be considered as a deformed action on the underlying classical space. One can moreover expand the star product "formally" in powers of $\theta$, as in deformation quantization. This is very useful to improve the leading (semi-classical) description systematically by higher-order corrections in $\theta$. In the context of noncommutative gauge theories (which arise in particular in matrix models), this leads to the concept of a Seiberg-Witten map [12].

Going beyond the semi-classical limit, the existence of a quantization map implies in particular a generalized Poincare-Birkhoff-Witt (PBW) property, in the sense that there should be a basis of $\mathscr{A}$ organized e.g. as ordered polynomials in $X^{\mu}$ (times some cutoff function such as $e^{-x^{2}}$ ). This means essentially that these "independent generators" $X^{\mu}$ can be ordered in some standard way. Hence the dimension of $\mathscr{M}_{\theta}$ could be characterized by the minimal number of generators $X^{a}$ which generate $\mathscr{A}$, which are functionally independent and satisfy such a PBW property.

Embedded noncommutative spaces. We are now ready to understand the geometric meaning of "generic but smooth" configurations in the matrix model. The key is to interpret the matrices $X^{a}$ as quantization of the Cartesian embedding map of $\mathscr{M} \subset \mathbb{R}^{D}$, i.e.

$$
X^{a} \sim x^{a}: \mathscr{M} \hookrightarrow \mathbb{R}^{D}
$$

In particular, we can write

$$
\left[X^{a}, X^{b}\right] \sim i\left\{x^{a}, x^{b}\right\}=i \theta^{\mu v} \partial_{\mu} x^{a} \partial_{v} x^{b}
$$

in the semi-classical limit, where $\theta^{\mu v}$ is the Poisson tensor in some local coordinates on $\mathscr{M}$. With a little more effort $[4,3]$, one can now show that

$$
\square \phi \equiv\left[X^{a},\left[X^{b}, \phi\right]\right] \delta_{a b} \sim-\left\{X^{a},\left\{X^{b}, \phi\right\}\right\} \delta_{a b}=-e^{\sigma} \Delta_{G} \phi(x)
$$


for any matrix resp. function $\phi \in \mathscr{A} \sim \phi(x)$. Here $\Delta_{G}$ is the standard Laplace operator associated to the effective metric $G_{\mu \nu}$ defined as follows [4]

$$
\begin{aligned}
G^{\mu v}(x) & :=e^{-\sigma} \theta^{\mu \mu^{\prime}}(x) \theta^{v v^{\prime}}(x) g_{\mu^{\prime} v^{\prime}}(x) \\
g_{\mu v}(x) & :=\partial_{\mu} x^{a} \partial_{v} x^{b} \delta_{a b}, \\
e^{-(n-1) \sigma} & :=\frac{1}{\theta^{n}}\left|g_{\mu v}(x)\right|^{-\frac{1}{2}}, \quad \theta^{n}=\left|\theta^{\mu v}\right|^{1 / 2} .
\end{aligned}
$$

All of these are tensorial objects on $\mathscr{M}$, e.g. $g_{\mu v}(x)$ is the metric induced on $\mathscr{M} \subset \mathbb{R}^{D}$ via pull-back of $\delta_{a b}$. The normalization factor $e^{-\sigma}$ is determined uniquely such that

$$
\frac{1}{\theta^{n}}=\sqrt{\left|G_{\mu v}\right|} e^{-\sigma}
$$

except for $n=1$ which we exclude for simplicity. This provides the desired explicit description of the matrix geometry at the semi-classical level. Higher-order corrections could be computed as an expansion in $\theta$, in the spirit of deformation quantization. This generalizes the known results for spaces with additional symmetry such as the fuzzy sphere to the case of generic matrix geometries.

The easiest way to see (4.5) is to consider the action for a scalar field coupled to the matrix model background

$$
S[\varphi] \equiv-\operatorname{Tr}\left[X^{a}, \phi\right]\left[X^{b}, \phi\right] \delta_{a b} \sim \frac{1}{(2 \pi)^{n}} \int d^{2 n} x \sqrt{\left|G_{\mu \nu}\right|} G^{\mu v}(x) \partial_{\mu} \phi \partial_{\nu} \phi .
$$

Writing the lhs as $\operatorname{Tr} \phi \square \phi$, we obtain (4.5). Moreover, note that $\phi$ in this action can be viewed as additional (i.e. transversal) matrix component $\phi \equiv X^{D+1}$ in an extended matrix model. For the same reason, (4.10) is precisely the action which governs e.g. nonabelian scalar fields in the original matrix model, which arise as fluctuations of the transversal matrices on stacks of such backgrounds $X^{a} \otimes \mathbf{1}_{n}$, cf. (7.1). This implies that these nonabelian scalar fields are governed by the effective metric $G_{\mu \nu}$. Similarly, one can show that all fields which arise in the matrix model as fluctuations of the matrices around such a background (i.e. scalar fields, gauge fields and fermions) are governed by $G_{\mu \nu}$, possibly up to a conformal factor $\sim e^{\sigma}$. This means that $G_{\mu \nu}$ is the effective gravitational metric.

We note the following observations:

- Assume that $\operatorname{dim} \mathscr{M}=4$. Then $G_{\mu \nu}=g_{\mu \nu}$ if and only if the symplectic form

$$
\omega=\frac{1}{2} \theta_{\mu \nu}^{-1} d x^{\mu} d x^{v}
$$

is self-dual or anti-selfdual [3].

- There is a natural tensor

$$
\mathscr{J}_{\gamma}^{\eta}=e^{-\sigma / 2} \theta^{\eta \gamma^{\prime}} g_{\gamma^{\prime} \gamma}=-e^{\sigma / 2} G^{\eta \gamma^{\prime}} \theta_{\gamma^{\prime} \gamma}^{-1} .
$$

Then the effective metric can be written as

$$
G^{\mu v}=\mathscr{J}_{\rho}^{\mu} \mathscr{J}_{\rho^{\prime}}^{v} g^{\rho \rho^{\prime}}=-\left(\mathscr{J}^{2}\right)_{\rho}^{\mu} g^{\rho v} .
$$


In particular, $\mathscr{J}$ defines an almost-complex structure if and only if $G_{\mu \nu}=g_{\mu v}$, hence for (anti-)selfdual $\omega$. In that case, $(\mathscr{M}, \tilde{g}, \omega)$ defines an almost-Kähler structure on $\mathscr{M}$ where

$$
\tilde{g}_{\mu \nu}:=e^{-\sigma / 2} g_{\mu \nu} .
$$

- The matrix model is invariant under gauge transformations $X^{a} \rightarrow X^{a \prime}=U^{-1} X^{a} U$, which semi-classically correspond to symplectomorphisms $\Psi_{U}$ on $(\mathscr{M}, \omega)$. This can be viewed in terms of modified embeddings $x^{a \prime}=x^{a} \circ \Psi_{U}: \mathscr{M} \rightarrow \mathbb{R}^{D}$ with equivalent geometry.

- Matrix expressions such as $\left[X^{a}, X^{b}\right] \sim i \theta^{\mu v} \partial_{\mu} x^{a} \partial_{\nu} x^{b}$ should be viewed as (quantizations of) tensor fields on $\mathscr{M} \subset \mathbb{R}^{D}$, written in terms of Cartesian coordinates $a, b$ of the ambient space $\mathbb{R}^{D}$. Note that they are always tangential, because $\partial_{\nu} x^{b} \in T_{p} \mathscr{M}$. Using appropriate projectors on the tangential resp. normal bundles of $\mathscr{M}$, this can be used to derive matrix expressions which encode e.g. the intrinsic curvature of $\mathscr{M}$, cf. $[13,14]$. This is important for gravity.

\section{Realization of certain generic 4D geometries in matrix models}

In this section, we want to show how a large class of generic 4-dimensional geometries can be realized as NC branes in matrix models with $D=10$. This should eliminate any lingering doubts about the geometrical scope of the matrix model approach to gravity. One way to see this is as follows:

1. Consider some "reasonable" generic geometry $\left(\mathscr{M}^{4}, g_{\mu \nu}\right)$ with nice properties, as explained below.

2. Choose an embedding $\mathscr{M} \hookrightarrow \mathbb{R}^{D}$. This is in general not unique, and requires that $D$ is sufficiently large. Using classical embedding theorems [15], $D=10$ should be enough to embed generic physically relevant 4-dimensional geometries (at least locally).

3. Equip $\mathscr{M}$ with an (anti-)selfdual closed 2-form $\omega$. Notice that this means $d \omega=d \star_{g} \omega=0$, i.e. $\omega$ is a special solution of the free Maxwell equations on $\mathscr{M}$. Such a solution generically exists for mild assumptions on $\mathscr{M}$, for example by solving the corresponding boundary value problem with $\omega$ being (anti-)selfdual on the boundary or asymptotically ${ }^{5}$. The requirements in step 1) should ensure that this is possible. For asymptotically flat spaces, $\omega$ should be asymptotically constant in order to ensure that the dilaton $e^{-\sigma}$ is asymptotically constant. In the case of compact extra dimensions $\mathscr{M}^{4} \times K$, this requirement may be relaxed.

As explained above, it follows that $(\tilde{g}, \omega)(4.14)$ is almost-Kähler. Under mild assumptions, one can then show [17] that there exists a quantization (4.1) of the symplectic space $(\mathscr{M}, \omega)$ in terms of operators on a Hilbert space ${ }^{6}$.

In particular, we can define $X^{a}:=\mathscr{I}\left(x^{a}\right) \in \mathscr{A}$ to be the matrix obtained as quantization of $x^{a}$, so that

$$
X^{a} \sim x^{a}: \mathscr{M} \rightarrow \mathbb{R}^{D} .
$$

\footnotetext{
${ }^{5}$ However, it may happen that $\omega$ vanishes at certain locations, cf. [16]. This might be cured through compact extra dimensions.

${ }^{6}$ The use of the almost-Kähler structure may only be technical and should actually not be necessary.
} 
The effective metric on $\mathscr{M}$ is therefore $G_{\mu \nu}$ as explained above.

4. Since $\omega$ is (anti-)selfdual it follows that $G=g$, and we have indeed obtained a quantization of $(\mathscr{M}, g)$ in terms of a matrix geometry. In particular, the matrix Laplacian $\square$ will approximate $\Delta_{g}$ for low enough eigenvalues, and fluctuations of the matrix model around this background describe fields propagating on this effective geometry.

\section{Deformations of embedded NC spaces}

Assume that $X^{a} \sim x^{a}: \mathscr{M} \hookrightarrow \mathbb{R}^{D}$ describes some quantized embedded space as before. The important point which justifies the significance of this class of configurations is that is preserved by small deformations. Indeed, consider a small deformation $\tilde{X}^{a}=X^{a}+A^{a}$ by generic matrices $A^{a} \in \mathscr{A}$. By assumption, there is a local neighborhood for any point $p \in \mathscr{M}$ where we can separate the matrices $X^{a}$ into independent coordinates and embedding functions,

$$
X^{a}=\left(X^{\mu}, \phi^{i}\left(X^{\mu}\right)\right)
$$

such that the $X^{\mu}$ generate the full ${ }^{7}$ matrix algebra $\mathscr{A}$. Therefore we can write in particular $A^{a}=$ $A^{a}\left(X^{\mu}\right)$, and assume that it is smooth (otherwise the deformation will be suppressed by the action). We can now consider $\tilde{X}^{\mu}=X^{\mu}+A^{\mu} \sim \tilde{x}^{\mu}\left(x^{\nu}\right)$ as new coordinates with modified Poisson structure $\left[\tilde{X}^{\mu}, \tilde{X}^{v}\right] \sim i\left\{\tilde{x}^{\mu}, \tilde{x}^{v}\right\}$, and $\tilde{\phi}^{i}=\phi^{i}+A^{i} \sim \tilde{\phi}^{i}\left(\tilde{x}^{\mu}\right)$ as modified embedding of $\tilde{\mathscr{M}} \hookrightarrow \mathbb{R}^{D}$. Therefore $\tilde{X}^{a}$ describes again a quantized embedded space. This property should also ensure that embedded NC spaces play a dominant role in the path integral (2.15).

If we do not want to assume the existence of a quantized embedded space, things are more difficult. The existence of a PBW property for a subset $X^{\mu}$ of the matrices might be a substitute, so that general functions $\phi\left(X^{\mu}\right)$ can be expanded in some basis of "ordered" functions of $X^{\mu}$, in particular $\left[X^{\mu}, X^{\nu}\right]=i \theta^{\mu \nu}\left(X^{\rho}\right)$. This should essentially imply the existence of some sort of quantization map (4.1). However this seems not very "intrinsic".

One particularly interesting point is the notion of dimension, which should be the number of independent generators in (6.1), or the rank of $\left[X^{a}, X^{b}\right]$. Semi-classically, this dimension can be extracted purely algebraically from $J_{b}^{a}:=-i\left[X^{a}, X^{c}\right] \delta_{c b}$, which semi-classically reduces to the tensor field $e^{\sigma / 2} \mathscr{J}_{v}^{\mu}$ (4.12). Therefore it satisfies a characteristic equation of order $\operatorname{dim} \mathscr{M}$ [3]. However it is not clear if this still holds e.g. for higher-order corrections in $\theta$. If so, this would provide a very useful intrinsic characterization of matrix geometry.

To obtain an intuition and to understand the meaning of "local description", consider the example of the fuzzy sphere. For example, we can solve for $X^{3}= \pm \sqrt{1-\left(X^{1}\right)^{2}-\left(X^{2}\right)^{2}}$, and use $X^{1}, X^{2}$ as local coordinate near the north pole $X^{3}=+1$ resp. the south pole $X^{3}=-1$. Each branch of the solution makes sense provided some restriction on the spectrum of $X^{3}$ is imposed, and in general "locality" might be phrased as a condition on the spectrum of some coordinate(s). Then the $X^{1}, X^{2}$ "locally" generate the full matrix algebra $\mathscr{A}$, and satisfy a PBW property.

The existence of a splitting (6.1) can be exploited further using the $I S O(D)$ symmetry of YangMills matrix models. In the semi-classical picture, one can thus assume for any given point $p \in \mathscr{M}$

\footnotetext{
${ }^{7}$ In topologically non-trivial situations they will individually generate only "almost" the full $\mathscr{A}$, and $\mathscr{A}$ is recovered by combining various such local descriptions. This will become more clear in the example of $S_{N}^{2}$.
} 
that $\partial_{\mu} \phi^{i}=0$, i.e. the tangent space is spanned by the first $d$ coordinates in $\mathbb{R}^{D}$. Moreover, $p$ can be moved to the origin using the $D$-dimensional translations. Then the matrix geometry looks locally exactly like $\mathbb{R}_{\theta}^{d}$, which is deformed geometrically by non-trivial $\phi^{i}\left(X^{\mu}\right)$ and a non-trivial commutator $\left[X^{\mu}, X^{v}\right]=i\left(\bar{\theta}^{\mu v}+\delta \theta^{\mu v}\left(X^{\alpha}\right)\right)$. These $X^{\mu}$ define "local embedding coordinates", which are analogous to Riemannian normal coordinates. Hence any deformation of $\mathbb{R}_{\theta}^{d}$ gives a matrix geometry as considered here, and vice versa any matrix geometry which is in some sense locally smooth should have such a local description. This completes the (heuristic) justification of our treatment of matrix geometry.

Finally, we are free in principle to use any other noncommutative "local coordinates", i.e. $Y^{\mu}\left(X^{v}\right)$, and write the resulting action in terms of $Y^{\mu}$. In the infinite-dimensional case, one can in particular try to choose ${ }^{8}$ the analog of local Darboux coordinates, defined as $\left[Y^{\mu}, Y^{v}\right]=i \bar{\theta}^{\mu v}$ for constant $\bar{\theta}^{\mu v}$. This amounts to the Moyal-Weyl quantum plane. However, the action then takes a highly non-trivial non-polynomial form ${ }^{9}$.

\section{Further aspects and generalizations}

Although we focused so far on matrix geometries which are quantizations of classical symplectic manifolds, it should be stressed again that matrix models are much richer and accommodate structures such as multiple branes, intersecting branes, manifolds suspended between branes, etc.

Recall that the algebra generated by (finite-dimensional) hermtitian matrices $X^{a}$ is always a product of simple matrix algebras, i.e. it decomposes into diagonal blocks. One particularly simple and important case is that of coinciding branes. Suppose that $X^{a} \in \mathscr{L}(\mathscr{H})$ is some matrix realization resp. quantization of $x^{a}: \mathscr{M} \hookrightarrow \mathbb{R}^{D}$ as discussed above. Then the following configuration

$$
Y^{a}=X^{a} \otimes \mathbf{1}_{n}=\left(\begin{array}{cccc}
X^{a} & 0 & 0 & 0 \\
0 & X^{a} & 0 & 0 \\
0 & 0 & \ddots & 0 \\
0 & 0 & 0 & X^{a}
\end{array}\right)
$$

should be interpreted as $n$ coinciding branes. This is instructive because the underlying algebra $\mathscr{A} \otimes \operatorname{Mat}(\mathrm{n}, \mathbb{C})$ can be interpreted in two apparently different but nonetheless equivalent ways: 1) as $s u(n)$ valued functions on $\mathscr{M}$ or 2) describing a higher-dimensional space $\mathscr{M} \times K$, where $\operatorname{Mat}(\mathrm{n}, \mathbb{C})$ is interpreted as quantization of some compact symplectic space $K$. Which of these two interpretations is physically correct depends on the actual matrix configuration, generalizing (7.1). Such extra dimensions provide a natural way of adding more structure to the effective physics such as physically relevant gauge groups etc., cf. [18].

Another variation of this idea allows to describe continuous superpositions of such branes, which involves a classical direction. For example, consider $Y^{a}=\left(X^{\mu} \otimes 1,1 \otimes C\right)$ where $C$ is a selfadjoint operator with continuous spectrum, such as a quantum mechanical position operator. This would describe the geometry of $\mathscr{M} \times \mathbb{R}$. However, in this case the matrix $X^{i}=1 \otimes C$ commutes with the $X^{\mu} \otimes 1$, and $\mathscr{M} \times \mathbb{R}$ is really a foliation with symplectic leaves $\{\mathscr{M} \times\{c\} ; c \in \mathbb{R}\}$ and

\footnotetext{
${ }^{8}$ this is clearly related to rigidity theorems for the Heisenberg algebra.

${ }^{9}$ I would like to thank Alexander Schenkel for discussions on this point.
} 
an extra classical direction. In that case, the effective metric $G^{\mu v}$ (4.6) in the matrix model is degenerate along the classical direction, so that fluctuations propagate only along the symplectic leaves. This is a very important property of the matrix model. It means that its effective geometry is necessarily non-commutative, justifying our focus on quantized symplectic spaces. This is also an important difference to the standard string theory picture where D-branes with a B-field do couple to the bulk physics. In our matrix model backgrounds, the bulk is essentially decoupled.

It is interesting to compare the present picture of embedded NC branes with other types of solutions of the IKKT model, such as the compactification on noncommutative tori in [19]. The latter are configurations of the type $U^{-1} X^{i} U=X^{i}+R^{i}$, which allow to obtain 10-dimensional compactified solutions such as $\mathbb{R}^{4} \times T^{6}$. These solutions do not belong to the class of embedded NC branes considered here. They are "space-filling" branes, whose tori are infinite-dimensional algebras which in some sense contains also a "winding" sector. They are not stable under small deformations (e.g. it makes an important difference whether $\theta$ is rational or irrational). In contrast, embedded NC spaces as considered here with extra dimensions such as (2.10) can be at most 8dimensional. They contain no winding modes and are stable under deformations. Therefore we are considering a different sector of the matrix model, which is better behaved in many ways.

Last but not least, it should be emphasized that the geometry and therefore gravity described by $X^{a}$ is not fixed but determined dynamically in these matrix models, depending notably on the presence of matter. The quantization of the IKKT model in terms of an integral over all (bosonic and fermionic) matrices (2.15) can be expected to be well-defined, because of maximal supersymmetry. Therefore this and related models provide excellent candidates for a quantum theory of gravity coupled to matter.

\subsection{Acknowledgments}

I would like to thank the organizers of the 2010 Corfu Summer Institute of elementary particle physics for providing a pleasant venue for stimulating and interesting discussions. I also want to thank Paolo Aschieri, who persistently confronted me with questions about basic aspects of the geometry in matrix models, which motivated this article. I also thank Clifford Taubes for pointing out limitations of the class of geometries under consideration, and Paul Schreivogel for useful discussions on the fuzzy torus. This work was supported by the FWF project P21610.

\section{References}

[1] N. Ishibashi, H. Kawai, Y. Kitazawa and A. Tsuchiya, "A large-N reduced model as superstring," Nucl. Phys. B 498 (1997) 467 [arXiv:hep-th/9612115]

[2] T. Banks, W. Fischler, S. H. Shenker and L. Susskind, "M theory as a matrix model: A conjecture," Phys. Rev. D 55 (1997) 5112 [arXiv:hep-th/9610043].

[3] H. Steinacker, "Emergent Geometry and Gravity from Matrix Models: an Introduction," Class. Quant. Grav. 27 (2010) 133001 [arXiv:1003.4134 [hep-th]].

[4] H. Steinacker, "Emergent Gravity and Noncommutative Branes from Yang-Mills Matrix Models," Nucl. Phys. B 810 (2009) 1 [arXiv:0806.2032 [hep-th]].

[5] J. Madore, “The Fuzzy sphere,” Class. Quant. Grav. 9 (1992) 69-88. 
[6] H. Grosse, J. Madore, H. Steinacker, "Field theory on the q deformed fuzzy sphere. 1.," J. Geom. Phys. 38 (2001) 308-342. [hep-th/0005273].

[7] A. P. Balachandran, S. Kurkcuoglu, S. Vaidya, "Lectures on fuzzy and fuzzy SUSY physics," Singapore, Singapore: World Scientific (2007) 191 p.. [hep-th/0511114].

[8] J. Hoppe, "Quantum theory of a massless relativistic surface and a two-dimensional bound state problem“, PH D thesis, MIT 1982; J. Hoppe,"Membranes and matrix models," [hep-th/0206192].

[9] P. B. Gilkey, "Invariance theory, the heat equation and the Atiyah-Singer index theorem,". CRC Press, 1995.

[10] A. Connes, "Gravity coupled with matter and the foundation of non-commutative geometry," Commun. Math. Phys. 182 (1996) 155 [arXiv:hep-th/9603053]; A. Connes, “A unitary invariant in Riemannian geometry,” Int. J. Geom. Meth. Mod. Phys. 5 (2008) 1215 [arXiv:0810.2091 [hep-th]].

[11] D. N. Blaschke, H. Steinacker, M. Wohlgenannt, "Heat kernel expansion and induced action for the matrix model Dirac operator,” [arXiv:1012.4344 [hep-th]].

[12] N. Seiberg, E. Witten, “String theory and noncommutative geometry,” JHEP 9909 (1999) 032. [hep-th/9908142].

[13] D. N. Blaschke, H. Steinacker, "Curvature and Gravity Actions for Matrix Models," Class. Quant. Grav. 27 (2010) 165010. [arXiv:1003.4132 [hep-th]]; D. N. Blaschke, H. Steinacker, "Curvature and Gravity Actions for Matrix Models II: The Case of general Poisson structure,” [arXiv:1007.2729 [hep-th]].

[14] J. Arnlind, J. Hoppe, G. Huisken, “Discrete curvature and the Gauss-Bonnet theorem," [arXiv:1001.2223 [math-ph]].

[15] C.J.S. Clarke, “On the Global Isometric Embedding of Pseudo-Riemannian Manifolds”, Proc. Royal Soc. London, A 314, 417 (1970); A. Friedman, J. Math. Mech. 10, 625 (1961)

[16] D. N. Blaschke, H. Steinacker, "Schwarzschild Geometry Emerging from Matrix Models," Class. Quant. Grav. 27 (2010) 185020. [arXiv:1005.0499 [hep-th]].

[17] D. Borthwick, A. Uribe, "Almost complex structures and geometric quantization“. Math. Res. Letters 3, 845-861 (1996); [arXiv:dg-ga/9608006]

[18] P. Aschieri, T. Grammatikopoulos, H. Steinacker and G. Zoupanos, "Dynamical generation of fuzzy extra dimensions, dimensional reduction and symmetry breaking," JHEP 0609 (2006) 026 [arXiv:hep-th/0606021]; H. Grosse, F. Lizzi and H. Steinacker, "Noncommutative gauge theory and symmetry breaking in matrix models," Phys. Rev. D 81 (2010) 085034 [arXiv:1001.2703 [hep-th]].

[19] A. Connes, M. R. Douglas, A. S. Schwarz, "Noncommutative geometry and matrix theory: Compactification on tori,” JHEP 9802 (1998) 003. [hep-th/9711162]. 